Real Analysis Exchange Vol. 22(1), 1996-97, pp. 390-395

D. N. Sarkhel, Mathematics Department, University of Kalyani, Kalyani 741 235, West Bengal, India

Rudolf Výborný, University of Queensland, 15 Rialanna, Kenmore, Qld

4069, Australia, e-mail: rv@@axiom.maths.uq.oz.au

\title{
A CHANGE OF VARIABLES THEOREM FOR THE RIEMANN INTEGRAL
}

\begin{abstract}
The change of variables formula for the Riemann integral is discussed and a theorem is proved which perhaps compares favorably with its counterpart in Lebesgue theory.
\end{abstract}

\section{Introduction}

It is often convenient to rewrite the usual formula for the change of variable

$$
\int_{G(a)}^{G(b)} f=\int_{a}^{b} f \circ G \cdot G^{\prime}
$$

in the form

$$
\int_{G(a)}^{G(b)} f=\int_{a}^{b} f \circ G \cdot g
$$

with $G(t)=G(a)+\int_{a}^{t} g$. Throughout this paper we shall assume that $g$ is Riemann integrable on $[a, b]$ and $G$ its Riemann primitive. According to Hobson [2, p. 508] Lebesgue as far back as in 1909 proved (1) for a monotone $G$ and R-integrable $f$. In this paper we show that (1) holds as long the integrand on either side of (1) is Riemann integrable. Our aim is also to give proofs within the framework of Riemann theory and accessible to undergraduates. First we prove (1) for a monotone $G$.

Key Words: Riemann integration, substitution

Mathematical Reviews subject classification: Primary: 26A39, 26A42

Received by the editors February 14, 1996 


\section{Monotone Substitution}

We need a weak variant of Henstock's lemma for the Riemann integral which is an immediate consequence of the definition based on upper and lower sums. Given $\varepsilon>0$ there is a partition

$$
D \equiv a=t_{0}<t_{1}<\cdots<t_{n}=b
$$

such that

$$
\sum_{1}^{n}\left|G\left(t_{i}\right)-G\left(t_{i-1}\right)-g\left(\tau_{i}\right)\left(t_{i}-t_{i-1}\right)\right|<\varepsilon,
$$

for any set of $\tau_{i}$ with $t_{i-1} \leq \tau_{i} \leq t_{i}$. Moreover (3) persists for any refinement of $D$.

Theorem 1 If $f$ is bounded on the range of $G$ and $g \geq 0$ on $[a, b]$ then

$$
\begin{aligned}
& \int_{G(a)}^{\overline{G(b)}} f=\int_{a}^{\bar{b}} f \circ G \cdot g, \\
& \int_{\underline{G(a)}}^{G(b)} f=\int_{\underline{a}}^{b} f \circ G \cdot g .
\end{aligned}
$$

If either $g \geq 0$ or $g \leq 0$ then if one side of (1) exists as a Riemann integral so does the other and equality holds.

Proof. It suffices to prove (4), the relation for the lower integrals follows by using (4) on $-f$. The rest of the theorem follows from (4-5). Let $|f| \leq M^{f}$, $|g| \leq M^{g}$, denote by $M_{i}$ and $\tilde{M}_{i}$ the least upper bound of $f \circ G$ and $f \circ G \cdot g$, respectively, on the interval $\left[t_{i-1}, t_{i}\right]$. We choose a partition (2) such that

$$
\sum_{1}^{n} \tilde{M}_{i}\left(t_{i}-t_{i-1}\right) \leq \int_{a}^{\bar{b}} f \circ G \cdot g+\varepsilon
$$


and (3) hold simultaneously. On every $\left[t_{i-1}, t_{i}\right]$ we find $\tau_{i}$ such that $M_{i}-\varepsilon<$ $f\left(G\left(\tau_{i}\right)\right)$, denote $x_{i}=G\left(t_{i}\right)^{1}$ and have

$$
\begin{aligned}
\int_{G(a)}^{\overline{G(b)}} f & \leq \sum_{1}^{n} M_{i}\left(x_{i}-x_{i-1}\right)=\sum_{1}^{n} M_{i}\left[G\left(t_{i}\right)-G\left(t_{i-1}\right)\right] \\
& \leq \sum_{1}^{n} M_{i} g\left(\tau_{i}\right)\left(t_{i}-t_{i-1}\right)+\sum_{1}^{n} M_{i}\left[G\left(t_{i}\right)-G\left(t_{i-1}\right)-g\left(\tau_{i}\right)\left(t_{i}-t_{i-1}\right)\right] \\
& \leq \sum_{1}^{n}\left[f\left(G\left(\tau_{i}\right)\right)+\varepsilon\right] g\left(\tau_{i}\right)\left(t_{i}-t_{i-1}\right)+M^{f} \varepsilon \\
& \leq \sum_{1}^{n}\left[f\left(G\left(\tau_{i}\right)\right)\right] g\left(\tau_{i}\right)\left(t_{i}-t_{i-1}\right)+\left(M^{f}+M^{g}(b-a)\right) \varepsilon \\
& \leq \sum_{1}^{n} \tilde{M}_{i}\left(t_{i}-t_{i-1}\right)+\left(M^{f}+M^{g}(b-a)\right) \varepsilon \\
& \leq \int_{a}^{\bar{b}} f \circ G \cdot g+\left(M^{f}+M^{g}(b-a)+1\right) \varepsilon .
\end{aligned}
$$

Consequently we have $(4)$ with $=$ replaced by $\leq$. For proving the reversed inequality we find a partition (2) such that

$$
\sum_{1}^{n} M_{i}\left[G\left(t_{i}\right)-G\left(t_{i-1}\right)\right] \leq \int_{G(a)}^{\overline{G(b)}} f+\varepsilon
$$

and (3) hold simultaneously. On $\left[t_{i-1}, t_{i}\right]$ we choose $\tau_{i}$ such that $\tilde{M}_{i}<f \circ G$. $g\left(\tau_{i}\right)+\varepsilon$. Then we have

$$
\begin{aligned}
\int_{a}^{\bar{b}} f \circ G \cdot g \leq & \sum_{1}^{n} \tilde{M}_{i}\left(t_{i}-t_{i-1}\right) \leq \sum_{1}^{n} f\left(G\left(\tau_{i}\right)\right) g\left(\tau_{i}\right)\left(t_{i}-t_{i-1}\right)+\varepsilon(b-a) \\
\leq & \sum_{1}^{n} M_{i}\left[G\left(t_{i}\right)-G\left(t_{i-1}\right)\right]+\varepsilon(b-a) \\
& \quad+M^{f} \sum_{1}^{n}\left|G\left(t_{i}\right)-G\left(t_{i-1}\right)-g\left(\tau_{i}\right)\left(t_{i}-t_{i-1}\right)\right| \\
\leq & \int_{G(a)}^{\overline{G(b)}} f+\varepsilon\left(b-a+1+M^{f}\right) .
\end{aligned}
$$

\footnotetext{
${ }^{1}$ Not all $x_{i}$ need to be distinct but that does not affect the proof.
} 


\section{Substitution Merely an Integral}

In this situation the existence of the integral $\int_{G(a)}^{G(b)} f$ places no restriction on the behavior of $f$ outside $[G(a), G(b)]$ therefore it is natural to replace this assumption by integrability of $f$ on the range of $G$.

Theorem 2 The change of variables formula (1) is valid if either

(i) $f$ is Riemann integrable on the range of $G$, or

(ii) $f$ is bounded on the range of $G$ and the Riemann integral on the righthand side of (1) exists.

For the proof we need the following

Lemma 3 If $z$ is a Lipschitz function on $[a, b]$ and $z^{\prime} \leq 0$ almost everywhere then $z(b) \leq z(a)$. If $z$ is Lipschitz and $z^{\prime}=0$ almost everywhere then $z(b)=$ $z(a)$.

Proof. It suffices to prove the first part of the lemma. Let $K>0$ be a Lipschitz constant for $z$. Given $\varepsilon>0$, there exists a countable system of open disjoint intervals $J_{n}, n=1,2, \ldots$ covering the set where $z^{\prime}$ either does not exist or is positive and such that

$$
\sum_{1}^{\infty}\left|J_{n}\right|<\frac{\varepsilon}{2 K} .
$$

Since

$$
z^{\prime}<\frac{\varepsilon}{2(b-a)}
$$

on

$$
[a, b] \backslash \bigcup_{1}^{\infty} J_{n},
$$

it is easily seen that the least upper bound of all $x \in[a, b]$ such that

$$
z(x)-z(a) \leq \frac{\varepsilon(x-a)}{2(b-a)}+\sum_{1}^{\infty} K\left|J_{n} \cap(a, x)\right|
$$

is the number $b$, and consequently $z(b)-z(a) \leq \varepsilon$. 
Proof of Theorem 2. Let $E=\{x \in(a, b) ; g$ continuous at $x\}, E_{0}=\{x \in$ $E ; g(x)=0\}, E_{-}=\{x \in E ; g(x)<0\}, E_{+}=\{x \in E ; g(x)>0\}$. Assuming (i) let

$$
\begin{aligned}
F(t) & =\int_{G(a)}^{G(t)} f \\
L(t) & =\int_{\underline{a}}^{t} f \circ G g .
\end{aligned}
$$

On $E_{0}$ we have $F^{\prime}(t)=L^{\prime}(t)=0$ because $f$ is bounded, $G^{\prime}(t)=0$ and $g$ continuous at $t$ with $g(t)=0$. Let $t \in E_{+}$, there is a positive $\alpha$ such that $g>0$ on $[t-\alpha, t+\alpha]$. If $|h|<\alpha$ then

$$
F(t+h)-F(t)=L(t+h)-L(t)
$$

by Theorem 1 . It follows that

$$
(F-L)^{\prime}(t)=0
$$

for $t \in E_{+}$. Similarly for $t \in E_{-}$. Consequently (6) holds on $E$ and hence a.e. on $[a, b]$. By the Lemma with $z=F-L$ we obtain

$$
\int_{G(a)}^{G(b)} f=\int_{\underline{a}}^{b} f \circ G g .
$$

Similarly

$$
\int_{G(a)}^{G(b)} f=\int_{a}^{\bar{b}} f \circ G g .
$$

Consequently

$$
\int_{\underline{a}}^{b} f \circ G g=\int_{a}^{\bar{b}} f \circ G g=\int_{G(a)}^{G(b)} f .
$$

Assume (ii). Let

$$
\begin{aligned}
\mathcal{L}(t) & =\frac{\int_{G(a)}^{G(t)} f}{\mathcal{F}(t)}=\int_{a}^{t} f \circ G g .
\end{aligned}
$$

The first part of the proof applies mutas mutandis with $F, L$ replaced by $\mathcal{F}$, $\mathcal{L}$, respectively. 
The assumption that $f$ is bounded in (ii) seems undesirable but, in fact, is essential. If $f(1 / n)=n$ and $g(1 / n)=0$ for $n=1,2, \ldots$, and $f(x)=0$, $g(x)=1$ otherwise then the integral on the right hand side of (1) exists but the one on the left hand side does not when $a=0$ and $b=1$.

\section{Concluding Remarks}

Theorem 1 remains valid if Riemann integrability is replaced by Lebesgue or Perron integrability. On the other hand the counterpart (i) of Theorem 2 for L-integral is false. This is because a composition of two AC functions need not be AC. The analog of Theorem 2 is not valid for the Perron integral either. This follows from the Corollary on p. 104 in [3]. If both integrals in (1) exist as Lebesgue or Perron integral then equation (1) holds. For the Lebesgue integral this was established by de la Valée Poussin already in 1915, [6], see also [5] Corollary 8 , the proof (of a more general theorem) for the Perron integral is in Goodman [1]. Theorem 2 is perhaps one of the rare examples when a theorem naturally and generally formulated within the framework of a theory is valid for the Riemann but not for the Lebesgue integral.

\section{References}

[1] G. S. Goodman, N-functions and Integration by Substitution, Rend. Sem. Mat. Fis. Milano, 47 (1978), 124-134.

[2] E. W. Hobson, The Theory of Functions ..., Vol. 1, Dover, New York.

[3] K. Krzyżewski, On change of variable in the Denjoy-Perron integral I, Coll. Math, 9 (1962), 99-104.

[4] K. Krzyżewski, On change of variable in the Denjoy-Perron integral II, Coll. Math, 9 (1962), 317-323.

[5] J. Serrin and D. E. Varberg, A General Chain Rule for Derivatives and Change of Variables Formula for the Lebesgue Integral, The American Mathematical Monthly, 76 (1969), 514-520.

[6] de la Valée Poussin, Sur l'intégrale de Lebesgue, Trans. Amer. Math. Soc., 16 (1915), 435-501. 